\title{
Association Between Contraceptive Use and Gestational Diabetes: Missouri Pregnancy Risk Assessment Monitoring System, 2007-2008
}

\author{
Brittney A. Kramer; Jeremy Kintzel, MA; Venkata Garikapaty, PhD \\ Suggested citation for this article: Kramer BA, Kintzel J, Garikapaty V. Association Between Contraceptive Use and \\ Gestational Diabetes: Missouri Pregnancy Risk Assessment Monitoring System, 2007-2008. Prev Chronic Dis \\ 2014;11:140059. DOI: http://dx.doi.org/10.5888/pcd11.140059圈.
}

PEER REVIEWED

\section{Abstract}

\section{Introduction}

The efficacy and safety of contraceptives have been questioned for decades; however, whether a relationship exists between hormonal contraceptives and gestational diabetes (GDM) is undetermined. The aim of this study was to investigate whether maternal risk for GDM was influenced by type of contraceptive method used before pregnancy.

\section{Methods}

Data collected in 2007 and 2008 by the Missouri Pregnancy Risk Assessment Monitoring System (PRAMS) were analyzed to determine if type of contraception before pregnancy influenced maternal risk for GDM. We used a logistic regression model to determine the adjusted odds for GDM given exposure to hormonal forms of contraception.

\section{Results}

Of the 2,741 women who completed the 2007-2008 PRAMS survey, 8.3\% were diagnosed with gestational diabetes, and $17.9 \%$ of the respondents had used hormonal contraceptive methods. Women who used hormonal methods of birth control had higher odds for gestational diabetes (adjusted odds ratio [AOR] $=1.43$; 95\% confidence interval [CI], 1.32-1.55) than did women who used no contraception. A protective effect was also observed for women who had used barrier methods of contraception ( $\mathrm{AOR}=0.79 ; 95 \% \mathrm{CI}, 0.72-0.86)$.

\section{Conclusion}

Findings suggest there may be a relationship between type of contraceptive method and GDM. More research is needed to verify contraception as a potential risk factor for GDM.

\section{Introduction}

Over the years, concerns have been raised about the possible association between hormonal contraceptives and various chronic diseases, including cardiovascular disease, breast cancer, and metabolic dysfunction. However, little is known about hormonal contraceptive use and its role in the development of gestational diabetes (GDM). Research has established a relationship between oral, hormonal contraceptive use and increased levels of serum glucose, insulin, and altered lipid profiles (1-4); however, much of the research is still unable to reach consensus on the long-term effects of contraceptives and an increased risk for GDM. Studies have examined the effects of contraception on the metabolisms of women who were previously diagnosed with GDM and found that women using hormonal methods of contraception had a higher risk for type 2 diabetes than did women using nonhormonal contraceptive methods (4). Nevertheless, no studies have investigated the effects of hormonal contraceptive use before pregnancy and the risk for GDM.

GDM is often diagnosed in weeks 24 through 28 of pregnancy and is characterized by no previous history of diabetes (type 1 or type 2) and high blood glucose levels (5). If left uncontrolled, GDM can result in macrosomia ("fat" baby), which can cause complications at birth and potentially force the mother to have a caesarian delivery. Other potential side effects involve preeclampsia and hypoglycemia (low blood glucose) in the mother, jaundice in the baby, and an increased risk for type 2 diabetes in both the mother and child later in life. GDM is estimated to affect $2 \%$ to $10 \%$ of all 
pregnancies, although US prevalence has increased since the late 1980 s and has more than doubled since $1990(6,7)$. Therefore, it is crucial to identify and understand risk factors for GDM to better tailor preventive and intervention measures.

The Missouri Pregnancy Risk Assessment Monitoring System (PRAMS) is an ongoing, population-based survey designed to identify and monitor select maternal experiences, attitudes, and behaviors that occur before, during, and shortly after pregnancy among women delivering a live infant. Data from PRAMS can be used to determine whether a relationship exists between a woman's contraceptive use before pregnancy and the development of GDM.

Research conducted by Visser et al compared hormonal and nonhormonal contraceptive use by diabetic women and found that high-dose oral contraceptives impaired glucose homeostasis (8). Likewise, Diab and Zaki found that fasting blood glucose was higher among users of oral contraceptives and Depo-Provera (depot-medroxyprogesterone acetate, DMPA), a progestogen-only reversible hormonal contraceptive injected every 3 months (9). After reviewing these studies, we hypothesized that an increased risk for GDM would be observed among women who had used hormonal methods of contraception. Our objective was to identify whether, after controlling for other factors, women who used hormonal methods of contraceptives before their pregnancy were more or less likely to develop GDM.

\section{Methods}

\section{Data collection}

PRAMS is conducted by the Missouri Department of Health and Senior Services as part of the national program sponsored by the Centers for Disease Control and Prevention (CDC). PRAMS is administered in 40 states and in New York City and represents approximately $78 \%$ of all US live births. Missouri began administering PRAMS in 2007. PRAMS collects information from a sample of women whose names are drawn from recent birth certificates (2 to 4 months after birth). A survey is mailed to each woman with telephone follow-up to those who do not respond. Responses are weighted to represent all live births in a given year. In Missouri, 2,741 women responded to the survey in 2007-2008, for an average weighted response rate of $63.9 \%$.

\section{Study population}

Missouri has participated in PRAMS continuously since 2007, beginning with Phase V of the survey (2007-2008). Missouri is collecting Phase VII data (2012 to present); we used Phase V data because the Phase V questionnaire asked participating women to identify the type of contraceptive they were using prepregnancy and postpartum, if applicable. Women could respond that they or their partner were using tubal ligation, vasectomy, birth control pills, condoms, injections (monthly or every 3 months), contraceptive patches, a diaphragm, a cervical ring, an intrauterine device, the rhythm method, withdrawal, or another method. They could also respond that they were not sexually active.

Women were identified as having been diagnosed with GDM if they reported that they had high blood glucose (diabetes) that started during the current pregnancy or if they had reported GDM on the matched birth certificate and did not respond in PRAMS that they had high blood glucose (diabetes) that started before the current pregnancy. This approach replicates GDM prevalence reported for Missouri by CDC's PRAMS C-PONDER query tool for 2007; 2008 data are unavailable in C-PONDER because Missouri did not meet the minimum required weighted response rate threshold for inclusion of $70 \%$.

Methods of prepregnancy contraception were categorized as hormonal, barrier, fertility awareness, other, or none. Methods of hormonal contraception included the pill, injections (received monthly or every 3 months), contraceptive patches, cervical ring, or intrauterine devices (IUD). Barrier methods included condoms or diaphragm, and fertility awareness methods included the rhythm or withdrawal methods. Respondents who reported that they were not using birth control when they became pregnant or reported that they were not using any of these specified methods were categorized as not using any contraception. All others were categorized as "other." These categories were selected on the basis of CDC categorization of birth control methods as either reversible birth control methods or permanent methods of birth control. All permanent methods (tubal ligation or vasectomy) were excluded because conception is next to impossible after these procedures, and the respondents were reporting this method as their contraceptive method before their most recent pregnancy.

\section{Data analysis}

Data were analyzed using SAS Enterprise Guide 4.3 (SAS Institute, Inc, Cary, North Carolina). A regression model was constructed with GDM as the outcome variable and other variables as predictor variables, representing respondents' demographics and preconception health, pregnancy health, and other birth outcomes. These variables included the mother's age, education level, race, Medicaid status (at delivery), marital status, contraceptive method, pregnancy intent, prepregnancy body mass index (BMI in $\mathrm{kg} / \mathrm{m}^{2}$, calculated from participants' self-reported height and weight), pregnancy weight gain, income, folic acid multivitamin use, adequacy of prenatal care, number of prenatal stressors experienced, use of assisted reproductive technology or fertility drugs, preterm or low birth weight baby, WIC 
participation, and a range of other prenatal maternal morbidities, including vaginal bleeding, high blood pressure, and preterm labor.

All variables from the univariate model were selected as predictor variables for the final, multivariate model. A backward stepwise selection was performed on all variables, which included respondents' age, education level, race, Medicaid status, marital status, pregnancy intent, contraceptive method, prepregnancy BMI, income, folic acid multivitamin use, and adequacy of prenatal care. For the adjusted multivariate model, methods of contraception were categorized and analyzed as hormonal, barrier, fertility awareness, other, or none. The final model was tested for collinearity, and all predictor variables were significant at $P<.001$.

\section{Results}

Of the 2,741 women who completed the 2007-2008 PRAMS, 8.3\% reported having been diagnosed with GDM in their most recent pregnancy. The most prevalent form of contraception used by Missouri PRAMS respondents was hormonal methods of contraception (17.9\%), followed by barrier methods (17.2\%), fertility awareness/rhythm method (6.8\%), and other (2.3\%). Of the total sample, $56 \%$ reported using no contraception.

Results of multivariate logistic regression indicated that women who used hormonal birth control methods were 1.4 times more likely to develop GDM than were those who did not use any birth control method ( $95 \%$ confidence interval [CI], 1.32-1.55, $P<$.001) (Table). Several other variables were associated with higher odds for developing GDM: women aged 30 or older compared with those younger than $20(\mathrm{AOR}=1.5 \mathrm{O} ; 95 \% \mathrm{CI}, 1.34-1.67 ; P<.001)$, women who received adequate prenatal care compared with women who received inadequate or intermediate prenatal care $(\mathrm{AOR}=$ 2.36; 95\% CI, 2.16-2.58), women enrolled in Medicaid at delivery compared with women who were not enrolled in Medicaid at delivery ( $\mathrm{AOR}=2.58 ; 95 \% \mathrm{CI}, 2.36-2.81$ ), women who identified as nonwhite or nonblack compared with white women $(\mathrm{AOR}=5.54 ; 95 \% \mathrm{CI}, 4.90-6.25)$, and women who were overweight or obese before pregnancy compared with women with a normal BMI before pregnancy ( $\mathrm{AOR}=3.04 ; 95 \% \mathrm{CI}, 2.84-3.24)$ (Table). A protective effect for GDM was observed for women who had used barrier methods for contraception compared with women who did not use any contraceptive methods (AOR $=0.79 ; 95 \% \mathrm{CI}, 0.72-0.86)$ (Table) and for women with an unintended pregnancy compared with women with an intended pregnancy (AOR $=0.39 ; 95 \% \mathrm{CI}, 0.37-0.42)$ (Table).

\section{Discussion}

This study provides evidence that hormonal contraceptive methods may increase a woman's risk for GDM in her following pregnancy, even after adjusting for maternal age, race, education and income level, marital status, Medicaid status at delivery, and type of prenatal care received. Other variables that were associated with higher odds for GDM were women who had a high school education or more, were 30 years or older, were of nonwhite or nonblack race, and were overweight or obese before pregnancy. These findings are consistent with those of other studies, one of which found that risk for GDM was correlated with increasing maternal age, high prepregnancy BMI, and high weight gain during pregnancy (10). Similarly, another study found that woman from racial/ethnic minority groups had a higher prevalence of GDM and that age and high BMI were also independent risk factors (11). It has been long accepted in the medical field that higher BMI, increasing age (>25y), and nonwhite race are risk factors for GDM (12). Our study, along with others, validates this consensus and suggests that intervention strategies should continue to focus on these demographic groups.

Other variables associated with higher odds for GDM included adequate access to prenatal care and being enrolled in Medicaid at delivery. Women who are enrolled in Medicaid and are receiving adequate prenatal care are more likely to see a doctor more frequently during their pregnancy and therefore have any pregnancy complications, including GDM, observed and diagnosed. This observation was also determined by Alexander and Cornely, who found that women who received intensive prenatal care were more likely to have more pregnancy complications, but also the most preferable birth outcomes (13). Furthermore, Griffin and colleagues determined that Medicaid expansion programs allowed women to receive the same treatment and prevention services on par with adequate prenatal care (14). These studies explain the higher odds observed for women who are receiving adequate prenatal care or Medicaid services; they are more likely to be diagnosed with GDM because they are more likely to be receiving frequent adequate care and therefore to be screened and diagnosed with it as opposed to women who may not be able to receive adequate care and consequently miss the opportunity to be diagnosed with GDM.

Protective effects were seen in women who had not intended or planned their pregnancy or who used barrier methods for contraception. As described previously, women who do not plan their pregnancy often underuse prenatal care services and receive inadequate care (15). This effect is the opposite of what was described previously, since women with an unintended pregnancy often inadequately seek and use prenatal care, so they are less likely to be seen and diagnosed by a doctor for GDM. On the basis of our hypothesis, women who are not using any form of hormonal contraception should be at an observed lower risk for developing GDM. Women who practice barrier methods of 
contraception are not increasing levels of hormones in their bodies and do not have the potential and undetermined side effects and, we propose, have a lower risk for GDM.

PRAMS data are subject to several limitations. First, PRAMS is a self-reported survey administered 2 to 6 months after the birth of a child, and results may be subject to recall bias. Second, weighting may not completely adjust for bias resulting from nonresponse. Third, PRAMS surveys are sent to a sample of women who delivered live babies in Missouri, and data are not generalizable to pregnant women with other outcomes or in different states.

This is the first study to evaluate the relationship between type of contraception used before pregnancy and risk for GDM. However, many studies have determined that hormonal methods of contraception have the potential to alter several homeostatic mechanisms including the hypothalamic pituitary axis as well as cortisol and carbohydrate metabolism. Burke suggested that prolonged exposure to high-dose estrogen treatment was associated with higher levels of unbound cortisol, which implied interference with cortisol homeostasis at the hypothalamic level (16). These higher levels of cortisol contribute to glucose intolerance and insulin resistance, which if left untreated can lead to diabetes (17).

This study's significant finding correlating contraceptive method and risk for GDM merits further exploration. The prevalence of GDM continues to increase worldwide, and definitive screening and preventive measures are needed to deter its chronic lifelong complications. Although researchers have not established a causal relationship between hormonal contraception use and GDM, results of our study suggest there may be an underlying correlating mechanism. More research is needed to assess hormonal contraception use as a potential risk factor for GDM.

\section{Acknowledgments}

The authors thank Mary Mosley, Missouri state PRAMS coordinator, for hosting this research project and making the data available to the authors and Leigh Tenkku for encouragement and continual help and support. No financial support was received for the analysis or support of this article.

\section{Author Information}

Corresponding Author: Venkata Garikapaty, PhD, Missouri Department of Health and Senior Services, Office of Epidemiology, Maternal and Child Health, 920 Wildwood Dr, P.O. Box 570, Jefferson City, MO 65102. Telephone: 573 -526-0452. E-mail: Venkata.Garikapaty@health.mo.gov.

Author Affiliations: Brittney A. Kramer, Jeremy Kintzel, Missouri Department of Health and Senior Services, Jefferson City, Missouri.

\section{References}

1. Eschwege E, Fontbonne A, , Simon D, Thibult N, Balkau B, Saint-Paul M, et al. Oral contraceptives, insulin resistance and ischemic vascular disease. Int J Gynaecol Obstet 1990;31(3):263-9. CrossRef 国 PubMed 圈

2. Godsland IF, Crook D, Simpson R, Proudler T, Felton C, Lees B, et al. The effects of different formulations of oral contraceptive agents on lipid and carbohydrate metabolism. N Engl J Med 1990;323(20):1375-81. CrossRef 圈 PubMed 圈

3. Russell-Briefel R, Ezzati TM, Perlman JA, Murphy RS. Impaired glucose tolerance in women using oral contraceptives: United States, 1976-1980. J Chronic Dis 1987;40(1):3-11. CrossRef 圈 PubMed 国

4. Kjos SL, Peters RK, Xiang A, Thomas D, Schaefer U, Buchanan TA. Contraception and the risk of type 2 diabetes mellitus in Latina women with prior gestational diabetes mellitus. JAMA 1998;280(6):533-8. CrossRef $\mathrm{E}$ PubMed 圈

5. Gestational Diabetes. American Diabetes Association. http://www.diabetes.org/are-you-at-risk/lower-yourrisk/gdm.html. Accessed January 3, 2014.

6. National diabetes statistics, 2011. National Diabetes Information Clearinghouse. http://diabetes.niddk.nih.gov/dm/pubs/statistics/\#fast. Accessed January 16, 2014.

7. Getahun D, Nath C, Ananth CV, Chavez MR, Smulian JC. Gestational diabetes in the United States: temporal trends 1989 through 2004. Am J Obstet Gynecol 2008;198(5):525-e1-5. CrossRef 国 PubMed 国

8. Visser J, Snel M, Van Vliet HA. Hormonal versus non-hormonal contraceptives in women with diabetes mellitus type 1 and 2. Cochrane Database Syst Rev 2006;(4):CDoo3990. PubMed F

9. Diab KM, Zaki MM. Contraception in diabetic women: comparative metabolic study of Norplant, depot medroxyprogesterone acetate, low dose oral contraceptive pill and CuT380A. J Obstet Gynaecol Res 2000;26 (1):17-26. CrossRef 圈 PubMed 圈 
Preventing Chronic Disease $\mid$ Association Between Contraceptive Use and Gestational Di... Page 5 of 6

10. Di Cianni G, Volpe L, Lencioni C, Miccoli R, Cuccuru I, Ghio A, et al. Prevalence and risk factors for gestational diabetes assessed by universal screening. Diabetes Res Clin Pract 2003;62(2):131-7. CrossRef 国 PubMed 国

11. Dornhorst A, Paterson CM, Nicholls JSD, Wadsworth J, Chiu DC, Elkeles RS, et al. High prevalence of gestational diabetes in women from ethnic minority groups. Diabet Med 1992;9(9):820-5. CrossRef 圈 PubMed 恩

12. Diseases and conditions: risk factors: gestational diabetes. Mayo Clinic. http://www.mayoclinic.org/diseasesconditions/gestational-diabetes/basics/risk-factors/con-2001485413. Accessed January 3, 2014.

13. Alexander GR, Cornely DA. Prenatal care utilization: its measurement and relationship to pregnancy outcome. Am J Prev Med 1987;3(5):243-53. PubMed 圈

14. Griffin JF, Hogan JW, Buechner JS, Leddy TM. The effect of a Medicaid managed care program on the adequacy of prenatal care utilization in Rhode Island. Am J Public Health 1999;89(4):497-501. CrossRef 圈 PubMed :

15. Delgado-Rodríguez M, Gómez-Olmedo M, Bueno-Cavanillas A, Gálvez-Vargas R. Unplanned pregnancy as a major determinant in inadequate use of prenatal care. Prev Med 1997;26(6):834-8. CrossRef $\mathrm{Q}$ PubMed $\mathrm{E}$

16. Burke CW. The effect of oral contraceptives on cortisol metabolism. J Clin Pathol 1969;1(1):11-8. CrossRef 园 PubMed 圈

17. Andrews RC, Walker BR. Glucocorticoids and insulin resistance: old hormones, new targets. Clin Sci 1999;96:513 -23. CrossRef圈 PubMed 圈

\section{Table}

Table. Maternal Characteristics and Odds of Developing Gestational Diabetes, Missouri Pregnancy Risk Assessment Monitoring System ( N = 2,741), 2007-2008a

\begin{tabular}{|c|c|c|}
\hline Characteristic & Prevalence & Adjusted Odds Ratio (95\% CI) \\
\hline \multicolumn{3}{|c|}{ Contraceptive method } \\
\hline Hormonal & 8.2 & $1.43(1.32-1.55)$ \\
\hline Barrier & 5.9 & $0.79(0.72-0.86)$ \\
\hline Fertility awareness & 10.0 & $1.38(1.24-1.53)$ \\
\hline Other & 12.0 & $0.59(0.47-0.73)$ \\
\hline None & 7.7 & 1 [Reference] \\
\hline \multicolumn{3}{|l|}{ Age, y } \\
\hline$<20$ & 4.9 & 1 [Reference] \\
\hline $20-29$ & 7.4 & $0.98(0.89-1.08)$ \\
\hline$\geq 30$ & 11.1 & $1.50(1.34-1.67)$ \\
\hline \multicolumn{3}{|l|}{ Education level } \\
\hline Less than high school & 7.2 & 1 [Reference] \\
\hline High school or more & 8.4 & $1.38(1.27-1.50)$ \\
\hline \multicolumn{3}{|l|}{ Race } \\
\hline White & 7.9 & 1 [Reference] \\
\hline Black & 7.7 & $1.32(1.22-1.43)$ \\
\hline Other & 18.0 & $5.54(4.90-6.25)$ \\
\hline \multicolumn{3}{|c|}{ Medicaid status at delivery } \\
\hline Medicaid & 9.0 & $2.58(2.36-2.81)$ \\
\hline Non-Medicaid & 7.4 & 1 [Reference] \\
\hline Pregnancy intent & & \\
\hline
\end{tabular}


Preventing Chronic Disease | Association Between Contraceptive Use and Gestational Di... Page 6 of 6

\begin{tabular}{|c|c|c|}
\hline Characteristic & Prevalence & Adjusted Odds Ratio (95\% CI) \\
\hline Unintended & 6.3 & $0.39(0.37-0.42)$ \\
\hline Intended & 9.6 & 1 [Reference] \\
\hline \multicolumn{3}{|c|}{ Prepregnancy weight status (BMI, kg/m²) } \\
\hline Underweight $(<18.5)$ & 2.3 & $0.79(0.64-0.97)$ \\
\hline Normal weight (18.5-24.9) & 4.8 & 1 [Reference] \\
\hline Overweight/obese $(\geq 25.0)$ & 11.5 & $3.04(2.84-3.24)$ \\
\hline \multicolumn{3}{|l|}{ Income, \$ } \\
\hline$<15,000$ & 7.3 & $0.57(0.51-0.64)$ \\
\hline $15,000-24,999$ & 9.3 & $0.73(0.65-0.82)$ \\
\hline $25,000-49,999$ & 9.2 & $0.72(0.66-0.80)$ \\
\hline$\geq 50,000$ & 7.8 & 1 [Reference] \\
\hline \multicolumn{3}{|l|}{ Folic acid multivitamin use } \\
\hline Use 4 times weekly & 8.1 & 1 [Reference] \\
\hline No use & 8.1 & $1.26(1.16-1.37)$ \\
\hline \multicolumn{3}{|l|}{ Prenatal care status } \\
\hline Adequate/plus & 9.3 & $2.36(2.16-2.58)$ \\
\hline Inadequate/intermediate & 4.4 & 1 [Reference] \\
\hline \multicolumn{3}{|l|}{ Marital status } \\
\hline Married & 8.5 & $0.83(0.77-0.89)$ \\
\hline Not married & 7.7 & 1 [Reference] \\
\hline
\end{tabular}

Abbreviation: CI, confidence interval; BMI, body mass index.

a All variables listed were included in final, multivariate model and found significant at $P<.001$

The opinions expressed by authors contributing to this journal do not necessarily reflect the opinions of the U.S.

Department of Health and Human Services, the Public Health Service, the Centers for Disease Control and Prevention, or the authors' affiliated institutions.

For Questions About This Article Contact pcdeditor@cdc.gov

Page last reviewed: July 17, 2014

Page last updated: July 17, 2014

Content source: National Center for Chronic Disease Prevention and Health Promotion

Centers for Disease Control and Prevention 1600 Clifton Rd. Atlanta, GA 30333, USA

800-CDC-INFO (800-232-4636) TTY: (888) 232-6348 - Contact CDC-INFO 